\title{
A multi-scenario Decision Support System for real-time operation of over-year multi-reservoir systems 2. DSS simulation
}

\author{
$\underline{\text { C. Arena }}^{\text {a }(\mathbb{D})}$, M. Cannarozzo a ${ }^{(1)}$, F. Oliva a ${ }^{\text {and M.R. Mazzola a }}{ }^{(\mathbb{D})}$ \\ ${ }^{a}$ Dipartimento di Ingegneria, Università degli Studi di Palermo, Palermo, Italy \\ Email: claudio.arena@unipa.it
}

\begin{abstract}
A companion paper (Arena et al, 2019) has introduced the architecture of the DSS and has described its governing equations. In a real-time, dynamic decision-making context, it is a tool to support decisions at the current time step concerning water allocations to municipal demand centres and irrigation districts as well as additional intakes from costly water sources in a water resources system featuring reservoirs with over-year behaviour. The DSS is designed as a linearized MIP (mixed integer programming) optimization model and as such, it includes an objective function and constraints on 1) mass balances at system's nodes, 2) systems' topology, 3) component's capacity, 4) spills, as well as non-empty conditions on reservoir storage at the end of the Forecasting Horizon (FH). It is a multi-scenario optimization tool because future, uncertain inflows are modelled, until the end of the current water year, as three different inflow scenarios: low flows, normal flows and high flows. The optimization model is solved for the three different scenarios and a unique solution that can be turned into one actual, implementable, decision at the present time step is obtained by imposing non-anticipatory (or congruity) constraints according to the principle of scenario aggregation (Rockfellar and Wets, 1987). The objective function is the weighted sum of the scarcity costs at all demand centres and of cost of water supply from additional sources along the multi-year FH, discounted to their present value, being the weights the occurrence probability of each of the three inflow scenarios. Given the over-year nature of the systems of interest for this study, the time unit is one month.
\end{abstract}

This paper first discusses estimation of scenario probabilities and of scenario inflows, then describes the application of the DSS to a real-world, two-reservoir system in Southern Italy. Its performances, in terms of scarcity costs and costs of additional, costly, water resources, are simulated over a forty-year historical period, on a monthly basis. Sensitivity of the DSS to different demand levels is explored considering different drift scenarios. Furthermore, in order to contrast the performances of the multi-scenario DSS presented here (DSSSC), we introduce a single-scenario DSS, identical to the multi-scenario one, except that decisions are made based on exogenous inflow forecast vectors. We look at two different types of forecast vectors that are meant to provide a lower and upper bound of DSS performances: the first type is a vector containing only the longterm means of monthly inflows and gives rise to DSS-WF (where WF stands for "worst forecast"). The second vector type contains instead real (i.e. actually occurred) inflows in the first six months of the FH and long-term means of monthly inflows for the remaining FH - 6 months. It gives rise to a DSS-BF (BF stands for "best forecasts").

Results show that DSS-SC compares quite favourably with DSS-BF: differences in total costs range from $37 \%$ for drift $=0.75$ to $27 \%$ for drift $=0.5$. DSS-BF clearly outperforms DSS-WF with improvements ranging from $17 \%(\mathrm{drift}=0.75)$ to $62 \%(\mathrm{drift}=0.90)$. In one demand condition, described by a drift of 0.9 , DSS-SC even outperforms DSS-BF. Investigation of this behaviour led to recognize that, at least for this drift, DSS-SC would manage the system so to keep the largest reservoir of the system full enough to allow issuing less restrictive irrigation supply reductions than its DSS-BF counterpart, and would therefore reduce the associated scarcity costs. This thought-provoking situation, if on the one hand confirms that in these long-memory systems "abrupt" failures can be the consequence of long-term policies and decision styles, on the other hand stimulates the reflection that DSS performances can indeed depend on a number of different factors that need to be investigated in deeper detail. From this standpoint, a single historic time series is probably not enough to explore the different possible behaviours of the DSS. For this reason, a stochastic validation of DSS-SC, by simulating its behaviour through synthetic time series, is in order and is the next research objective.

Keywords: Decision Support Systems, real time reservoir management, scenario optimization 
Arena et al., A multi-scenario Decision Support System for real-time operation of over-year multi-reservoir systems 2. DSS simulation

\section{INTRODUCTION}

The companion to this paper (Arena et al, 2019) has introduced the general concept of the DSS, its governing equations, as well as the general objectives of this study. In a real-time management context, the study aims at comparing the performances of a DSS based on a multiple-inflow scenario optimization model such as the one discussed here, and the same DSS based on a single-scenario model where inflows are provided in the form of exogenous forecasts. The companion paper also contains the methodologies followed to estimate scarcity costs (model's objective function consists of minimizing them together with the costs from the purchase of water from additional sources) and the methodologies adopted to linearize the model. In this paper we will first discuss some issues related to the estimation of inflows in the multi-scenario setting and will introduce the application of the DSS on a real two-reservoir system in Southern Italy. Finally, we will provide and discuss results from DSS simulation, contrasting multi-scenario model performances with those obtained from the same DSS in a single-scenario mode, working with exogenous inflow forecasts.

\section{ESTIMATION OF SCENARIO PROBABILITIES AND INFLOWS}

As explained in the companion paper, in the DSS, decisions at the current time step on water allocations and water intakes from additional costly water sources are made solving, for three different inflow scenarios "sc" (low, normal, high) an optimization model along a multi-year Forecasting Horizon (FH). The model includes the objective function and constraints on 1) mass balances at system's nodes, 2) systems' topology, 3) component's capacity, 4) spills, as well as non-empty conditions on reservoir storage at the end of the Forecasting Horizon (FH) (Arena et al, 2019). A unique solution that can be turned into one actual, implementable, decision is obtained by imposing non-anticipatory (or congruity) constraints according to the principle of scenario aggregation (Rockfellar and Wets, 1987). The objective function is the weighted sum of the scarcity costs at all demand centres and of cost of water supply from additional sources along the multiyear FH, discounted to their present value, being the weights the occurrence probability of each of the three inflow scenarios. Given the over-year nature of the systems of interest for this study, the time unit is one month.

The working assumption is that at each time step these three inflow scenarios depend on the extent of occurred inflows from the beginning of the current water year (September) to the beginning of the present month: the level of past cumulative inflows qualifies the present hydrological condition of the system as dry, normal or wet. The probabilities of each of the three inflow scenarios appearing in the objective function are assumed to coincide with the estimated transition probabilities from system's current state (dry, or normal or wet) to a $\operatorname{low}(\mathrm{sc}=1)$, normal $(\mathrm{sc}=2)$ and high $(\mathrm{sc}=3)$ condition of cumulative future inflows from the present month to the end of the water year.

To decide what cumulative inflows should be considered low, normal or high and to estimate transition probabilities, historical records of monthly inflows to system's reservoirs must be analyzed. In the application for this study, 45 yr. inflow series, from 1971 to 2016, were used. Since in the objective function of the model, scenario probabilities are given for the whole system, not for each single inflow series, the historical record to analyze is $I_{s y s(n)}$, the sum of inflows $I_{(i, n)} \mathrm{i}=1, \ldots \mathrm{N}_{r e s} ; \mathrm{n}=1 \ldots 540$, into the $N_{\text {res }}$ reservoirs of the system: $I_{s y s(n)}=\sum_{i=1}^{N r e s} I_{(i, n)}$. The series to analyze are Cumulative System Inflows from the beginning of current water year (September) until the end of current month $\mathrm{m} C I_{s y s(m)}=\sum_{\tau=1}^{m} I_{s y s}$ and Future System Inflows $F I_{s y s(m)}=\sum_{\tau=m+1}^{12} I_{s y s}(\tau)$

Figure 1 shows the relationship between month m, cumulative inflows $C I_{s y s(m)}$ and future inflows $F I_{s y s(m)}$ until the end of the water year.

Each 45-yr. sample of cumulative inflows (e.g. October to April) as well as each $45 \mathrm{yr}$. sample of future inflows (e.g. February to September), is assumed to be distributed according to a mixture $g_{\theta}(x)$ of low, normal and high inflows:

$$
g_{\theta}(x)=\sum_{j=1}^{3} \lambda_{j} \Phi_{j}(x)
$$

Where $x$ are inflows (one of the 23 different CI or FI inflow types), $\lambda_{j}$ identifies the "concentration" of each of the three components in the mixture: $\sum_{\mathrm{j}=1}^{3} \lambda_{\mathrm{j}}=1, \Phi_{\mathrm{j}}(x)$ is the distribution of the $\mathrm{j}$-th component (a mixture of normal distribution was selected here) and $\theta$ is the vector containing $\lambda_{j}$ and parameters $\mu_{\mathrm{j}}$ and $\sigma_{\mathrm{j}}$ of distributions 
Arena et al., A multi-scenario Decision Support System for real-time operation of over-year multi-reservoir systems 2. DSS simulation

$\Phi_{\mathrm{j}}(x)$

OCT-SEPT NOV-SEPT DEC-SEPT JAN-SEPT FEB-SEPT MAR-SEPT APR-SEPT MAY-SEPT JUN-SEPT JUL-SEPT AUG-SEPT SEPT

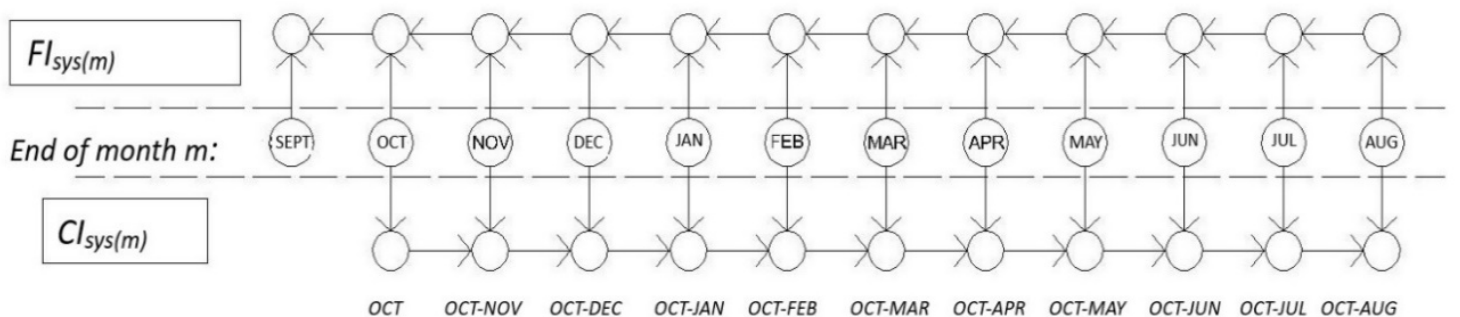

Figure 1. Relationship between month-types, cumulative inflow and future (scenario) inflows

Maximum likelihood estimation was performed through an EM (Expectation-Maximization) algorithm available in the "Mixtools" R-package (Benaglia et al. 2009). Figure 2 shows graphically the outcomes of the estimation procedure.
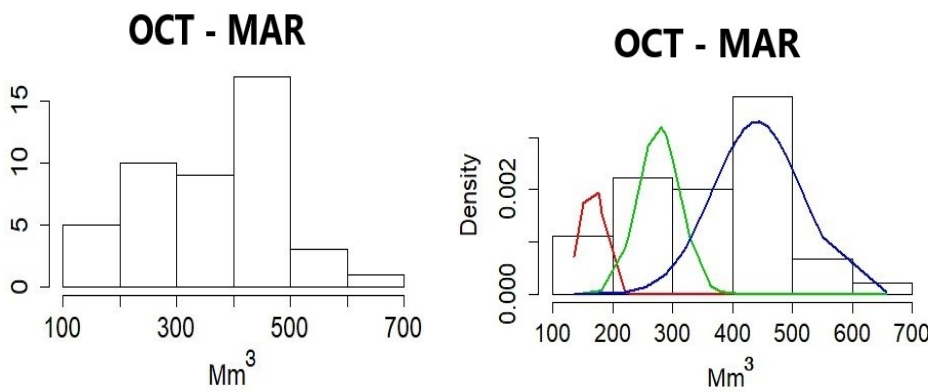

FEB - SEPT

FEB - SEPT
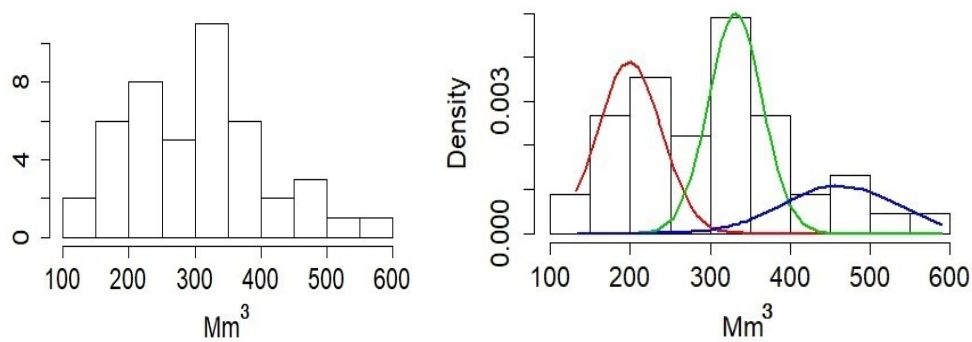

Figure 2. Examples of three-component mixture distributions (blue $=$ high, green $=$ normal, red $=$ low) fitted to 45 -year $C I_{s y s}$ and $F I_{s y s}$ samples

From the estimated $\lambda_{j}{ }^{(\mathrm{CI})}$ and $\lambda_{\mathrm{j}}{ }^{(\mathrm{FI})}$, one may identify in the inflow sequence ordered in ascending order the thresholds of low, normal and high flows. Transition probabilities, that are identified with probabilities sc of the single scenarios of future inflows, depend on the month-type, are defined by (2) and may be easily estimated from the historical records.

$$
S c_{(m, i, f)}=\operatorname{Prob}\left[F I_{s y s(m)} \text { is in state } f \mid C I_{s y s(m)} \text { is in state } i\right]
$$

\subsection{Determining scenario inflows}

Finally, the vector $\mathrm{I}_{\text {forecast }(i, t, s)}$ containing inflows from $\mathrm{t}=1$ to $\mathrm{t}=\mathrm{FH}$ ( $\mathrm{FH}$ is set equal to 24 in this application) can be calculated for each relevant site i (reservoir) and for each of three scenarios. This vector contains $12-$ $\mathrm{t}$ values deriving from the calculations explained below and $12+\mathrm{t}$ average monthly values. Vectors of inflow forecasts are built in this fashion for two reasons. The first is that a FH of 24 months was identified as optimal for the specific system under study, albeit using a different objective function (sum of standardized squared 
Arena et al., A multi-scenario Decision Support System for real-time operation of over-year multi-reservoir systems 2. DSS simulation

deviations in Arena et al. 2017). The second, and more significant for the objectives of this study, is that limiting scenario formulation only to the hydrologic year allows comparison of the multi-scenario model presented here with a single-scenario DSS using seasonal forecasts whose accuracy, at least at the state of the art, is very limited for periods longer than six months.

Calculations are performed by disaggregating in time (among months to the end of the water year) and space (among reservoirs) system's average "low", "normal" and "high" of future inflows $F I_{\text {sys }}$ estimated through the Mixtools package. This is done following the steps below:

1. For each reservoir assess the long-term means of monthly system's inflows reservoir $m_{(i, p),} \mathrm{p}=$ $1 \ldots .12 ; \mathrm{i}=1 \ldots N_{\text {res }}$

2. For each month-type $\mathrm{m}$, compute the ratios $R_{(i, p)}=m_{(i, p)} / \sum_{p=m}^{12} m_{(i, p)}$;

3. For each series of $F I_{s y s(m)}$, evaluate means $\mu_{\mathrm{j}}=\mu_{(\mathrm{sc})} \mathrm{j}=1 \ldots 3$ (low, normal or wet scenario) of the components of the mixture distribution;

4. To obtain the vectors of inflows into the i-th reservoir of the system introduce ratios $R_{(i, p)}=m_{(i, p)} / \sum_{i=1}^{N_{r e s}} m_{(i, p)}$;

5. For each month-type, obtain monthly system inflows until the end of the water year $I_{s y s(m, s c)}=$ $\left[R_{m} \cdot \mu_{(\mathrm{sc})}, R_{m+1} \cdot \mu_{(\mathrm{sc})} \ldots . R_{\text {Sept }} \cdot \mu_{(\mathrm{sc})}\right]$;

6. Complete the vector with mean monthly values $I_{\text {forecast }(i, m, s c)}=\left[R_{(i, m)}^{\prime} \cdot I_{s y s(m, s c)}, R_{(i, m+1)}^{\prime}\right.$. $\left.I_{\text {sys }(m+1, s c)} \ldots R_{(i, S e p t)}^{\prime} \cdot I_{s y s(S e p t, s c)}, m_{(\mathrm{i}, \mathrm{Oct})}, m_{(\mathrm{i}, \mathrm{Nov})}, m_{(\mathrm{i}, \mathrm{Dec})} \ldots . . m_{(\mathrm{i}, \mathrm{Sept})}, \ldots m_{(\mathrm{i}, \mathrm{m}-1)}\right]$.

\section{APPLICATION}

The DSS was tested on a real-world scheme in Southern Italy (Figure 3). Basic information on supply sources hydrology, demand and relevant model parameters are reported in Figure 3. The two sources of additional water are provided by the Salento Aquifer that supplies directly the demand centres of Salento, and by Cogliandrino reservoir, a reservoir for hydropower production that does not belong to the system, but that can provide additional water at the cost of lost hydropower production. Constraints on withdrawal from these two sources are indicated in Figure 3 in the form of a withdrawal threshold $\mathrm{V}_{\max }$. Unit prices for both these resources are set in this study at $0.15 € / \mathrm{m}^{3}$. Monthly target demands for municipal supply TDmun are assumed constant along the year, while monthly target demands for irrigation TDirr vary along the year, as per the values shown in Figure 3.The behaviour of the DSS was simulated over a 40-yr. period, from water year 1966/67 to $2005 / 2006$. Simulation consists in running the DSS using initial reservoir storages and inflow forecasts for the FH (two years ahead) at the first month (October) of water year 1966/67. The DSS then provides the schedule of water allocations to demand centres and of expenditures for additional resources for two years in advance. Obviously, only the first decision, the one for the current month, is implemented. In the next simulation step, actual inflows into the reservoirs in the past month become known, so that new initial reservoir storages can be calculated and the DSS is run again with updated reservoir storages and inflow forecasts. The process continues for the subsequent 479 time steps.

\subsection{The redistribution procedure}

In order to adjust for situations when planned decisions cannot be implemented because they are based on forecasts that have overestimated inflows and water stored is too little to enforce the planned decision, a redistribution procedure was added to the simulation step. It starts when at least one of the reservoirs is empty and is formulated as an optimization model to allocate resources only at the present time step where the issue arises. Constraints are the same as those of the DSS, except for equations on spill and on final storage at the end of the FH that are not necessary since no spill is expected to take place in any of the reservoir of the system when the redistribution procedure is activated. In addition, there is no constraint on final storage to enforce as the procedure is only applied to the current time step. In the redistribution procedure the objective function is the same as in the DSS except that no scenarios are considered since the objective here is the equilibrium among scarcity costs given the available resources. As this equilibrium is to be found only in the current time step, not along the entire FH, scarcity costs for irrigation cannot be evaluated on a yearly scale. To overcome this issue, scarcity costs are expressed as a linear function of water deficits, using as slope the slope of the third piece of the linearized scarcity cost-deficit relations (see Figure 3 of the companion paper). 
Arena et al., A multi-scenario Decision Support System for real-time operation of over-year multi-reservoir systems 2. DSS simulation

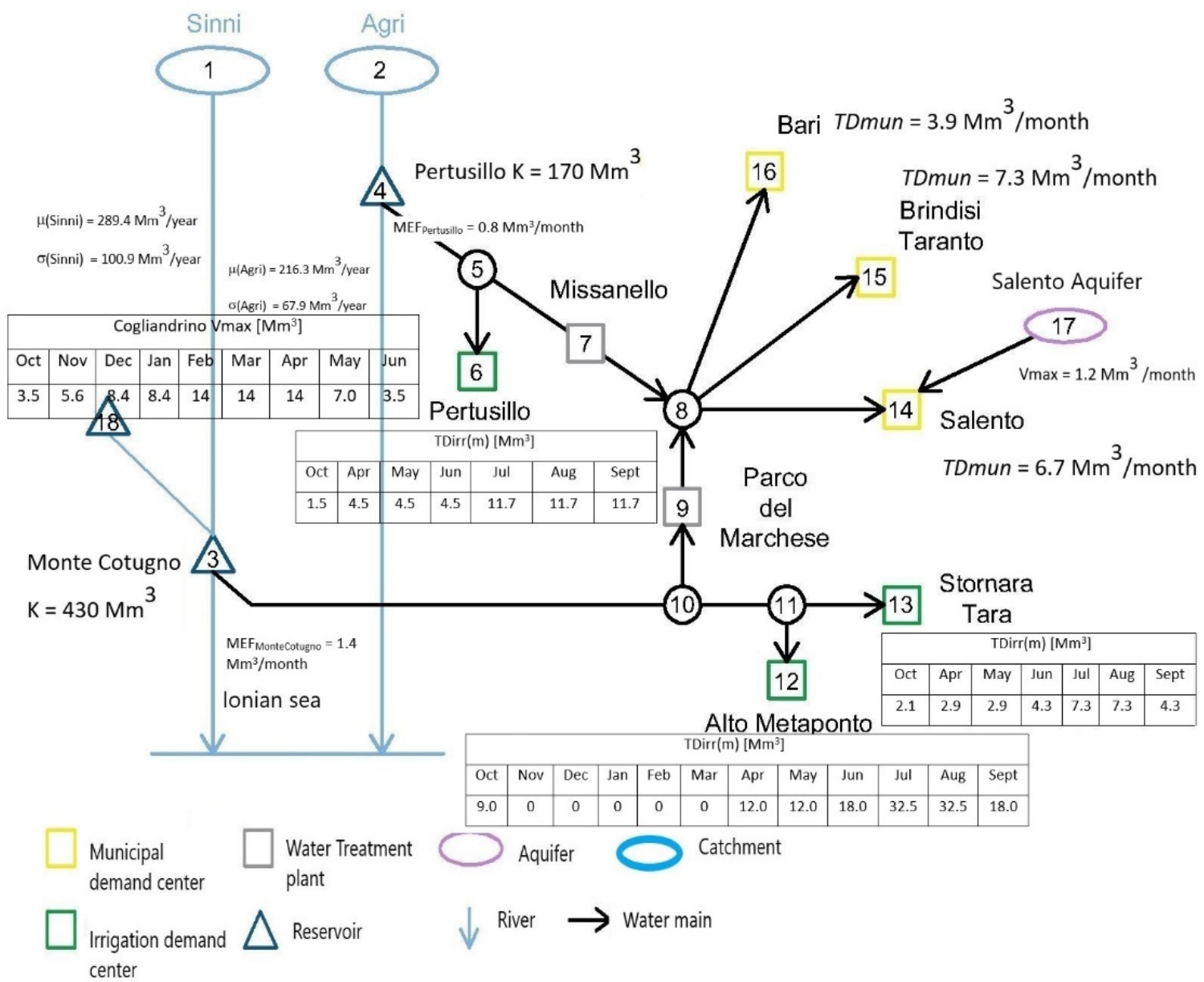

Figure 3. Schematic of the Agri-Sinni water resources system. Numbers indicate nodes in the model. Additional, costly water resources are withdrawn from node 18 (Cogliandrino reservoir) and Salento Aquifer (Node 17). Target demand values for municipal uses TDmun and for irrigation purposes (TDirr) are referred to drift $=0.75($ section 3.3$)$

\subsection{A refinement of the decision-making process}

Finally, in order to increase the verisimilitude of the simulated decision-making process, it was decided that the April schedule issued at the end of March for irrigation uses must remain unchanged along the forthcoming irrigation season, starting in April and finishing at the end October. This is consistent with the procedures presently in place, that see a centralized Authority allocating available water at the beginning of the irrigation season to the different reclamation consortia. During the irrigation season, however, the DSS continues to run because adjustments in municipal allocations are still allowed and because additional water may be purchased to benefit both municipal and irrigation demand centres. This refinement in the decision-making process requires some changes in the structure of the DSS (for instance, in the month-types belonging to the irrigation season the Objective Function need no longer include scarcity costs for irrigation in the current year, as allocations for irrigation in the current year are no longer decision variables). Details are contained in Oliva (2019).

\subsection{Boundary conditions}

In order to assess the performances of the DSS in different conditions, various boundary conditions in terms of supply (type of inflow forecasts) and demand were used. As far as the latter is concerned, we use the concept of drift (Vogel et al. 1999) to explore the behaviour of the DSS when the same system, supplied by the same inflows, is challenged by different demand levels. Drift is defined as $(\mu-Y) / \sigma$, where $\mu$ is mean annual inflow into the system, $\sigma$ its standard deviation and $Y$ the average annual yield. If inflow series are unchanged, lower drifts imply a higher pressure on water resources ( $\mathrm{Y}$ is closer to $\mu$ due to higher demand) while higher drift 
Arena et al., A multi-scenario Decision Support System for real-time operation of over-year multi-reservoir systems 2. DSS simulation

values indicate a lower pressure on water resources. Three different drifts were considered: 0.50, 0.75 and 0.90. Furthermore, in order to contrast the performances of the multi-scenario DSS presented here, we introduce a single-scenario DSS, identical to the multi-scenario one, except that decisions are made based on exogenous forecast vectors. We look at two different types of forecast vectors that are meant to provide a lower and upper bound of DSS performances. The first type consists of a vector containing only the long-term means of monthly inflows. The second type contains instead real (i.e. actually occurred) inflows in the first six months of the FH and long-term means of monthly inflows for the remaining eighteen months. These two single-scenario DSSs are supposed to describe the entire range of DSS performances because the DSS using average monthly inflows as forecasts is run with readily available, but poor inflow forecasts. At the other end, a perfect foresight for six months ahead should be considered as the upper limit of the quality of currently available seasonal forecast services.

\section{RESULTS AND DISCUSSION}

Table 2 contains average yearly municipal and irrigation scarcity costs, expenditures for additional water resources, and total costs over the 40-yr. period of simulation for drift $=0.5,0.75$ and 0.9 using the proposed multi-scenario DSS (SC) and single-scenario DSSs, one making use of long-term average inflows (DSS-WF Worst Forecast) and the other using six-month-ahead real inflows as forecast, complemented by 18 months of long-term average inflows (DSS-BF Best Forecast). Looking at total costs, for drift $=0.5$ and drift $=0.75$, costs resulting from system management supported by the multi-scenario DSS (DSS-SC) stay in the middle between DSS-WF and DSS-BF, being clearly closer to DSS-BF for drift $=0.5$ and staying in the middle between DSS$\mathrm{BF}$ and DSS-WF for drift $=0.75$. Quite surprisingly, for drift $=0.90$, DSS-SC even outperforms DSS-BF although in this case the magnitude of damages (scarcity costs) and of the related coping measures are clearly considerably lower than those materialized for lower drifts. The reason for this behaviour allows some insight into model's working and will be explained later. Sticking to Table 2, it should be also highlighted that the good performances of DSS-SC are achieved at a price: financial expenditures for additional water resources are highest for the DSS-SC for drift $=0.75$ and drift $=0.50$, and very close to DSS-BF for drift $=0.90$. However, low financial costs do not necessarily imply good management, as can be seen in the case of DSS-WF: low expenditures for additional resources will ultimately impose high social (scarcity) costs on final users.

Table 2. Comparison of simulated average annual costs for multi-scenario DSS-SC and for single-scenario DSS-BF and DSS-WF for the three drifts considered

\begin{tabular}{|c|c|c|c|c|c|c|c|c|c|c|c|c|}
\hline \multirow[t]{2}{*}{ Drift } & \multicolumn{3}{|c|}{$\begin{array}{c}\text { Municipal Scarcity } \\
\text { Cost [M€/year] }\end{array}$} & \multicolumn{3}{|c|}{$\begin{array}{c}\text { Irrigation scarcity Cost } \\
{[\mathrm{M} € / \text { year }]}\end{array}$} & \multicolumn{3}{|c|}{$\begin{array}{c}\text { Expenditures for } \\
\text { additional water } \\
\text { resources [M€/year] }\end{array}$} & \multicolumn{3}{|c|}{ Total Cost [M€/year] } \\
\hline & $\begin{array}{l}\text { DSS- } \\
\text { WF }\end{array}$ & $\begin{array}{l}\text { DSS- } \\
\text { BF }\end{array}$ & $\begin{array}{l}\text { DSS- } \\
\text { SC }\end{array}$ & $\begin{array}{l}\text { DSS- } \\
\text { WF }\end{array}$ & $\begin{array}{c}\text { DSS- } \\
\text { BF }\end{array}$ & $\begin{array}{l}\text { DSS- } \\
\text { SC }\end{array}$ & $\begin{array}{l}\text { DSS- } \\
\text { WF }\end{array}$ & $\begin{array}{c}\text { DSS- } \\
\text { BF }\end{array}$ & $\begin{array}{l}\text { DSS- } \\
\text { SC }\end{array}$ & $\begin{array}{l}\text { DSS- } \\
\text { WF }\end{array}$ & $\begin{array}{c}\text { DSS- } \\
\text { BF }\end{array}$ & $\begin{array}{l}\text { DSS- } \\
\text { SC }\end{array}$ \\
\hline 0,5 & 4,41 & 0,17 & 0,00 & 4,67 & 2,61 & 3,16 & 0,81 & 1,24 & 1,96 & 9,90 & 4,02 & 5,12 \\
\hline 0,75 & 2,58 & 0,36 & 0,40 & 2,57 & 1,99 & 2,63 & 0,43 & 1,04 & 1,62 & 5,59 & 3,40 & 4,65 \\
\hline 0,9 & 0,70 & 0,00 & 0,13 & 1,54 & 1,48 & 0,43 & 0,16 & 0,38 & 0,36 & 2,40 & 1,85 & 0,92 \\
\hline
\end{tabular}

Several elements can help understand why DSS-SC over-performs DSS-BF for drift $=0.90$. In the first place, as shown in Figure 5, higher total costs in DSS-BF are due to a cost peak at months 286 (a July) and 287 (an August) of the simulation period. This cost peak is associated to a deficit in irrigation centres at node 14 and especially node 12 , the one with the largest water demands in the system. These two demand centres are supplied by Monte Cotugno reservoir. An analysis of volumes stored in this reservoir in months 286 and 287 (Figure 6) indicates that management with DSS-SC makes available in those two months some $25 \mathrm{Mm}^{3} \mathrm{more}$ than those available following decisions provided by DSS-BF. In addition, it should not be forgotten that irrigation allocations for months from April to September are already fixed at the end of April (month 283 in Figure 6). In that month of April, storage at Monte Cotugno reservoir is quite low with DSS-BF and justifies issuing significant supply restrictions for the forthcoming season. Water storage with DSS-SC is still quite low, but allows DSS-SC issuing a less restrictive irrigation schedule, bringing to more restrained economic losses, as shown in Figure 5. This situation is not accidental: an examination of the frequency of storage levels in the two reservoirs of the system shows (Figures 7 and 8) that, at least for drift, $=0.9$ DSS-SC tends to exploit more resources from Pertusillo reservoir than from Monte Cotugno, keeping the latter fuller than done by DSS-BF. 


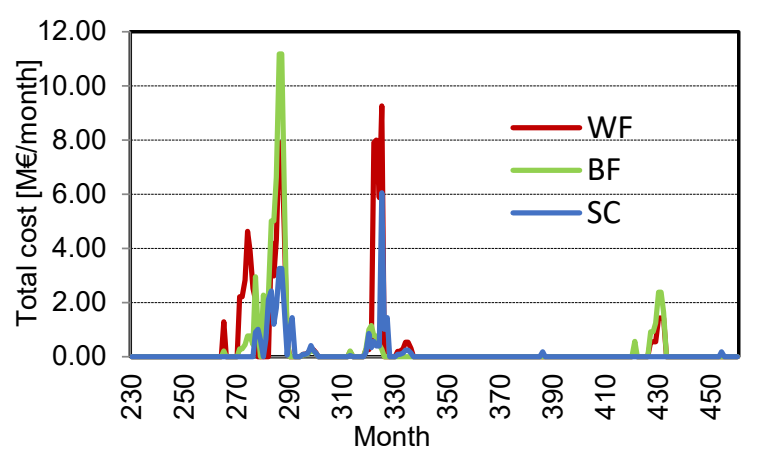

Figure 5. Pattern of total costs along deficit periods in the simulation horizon.

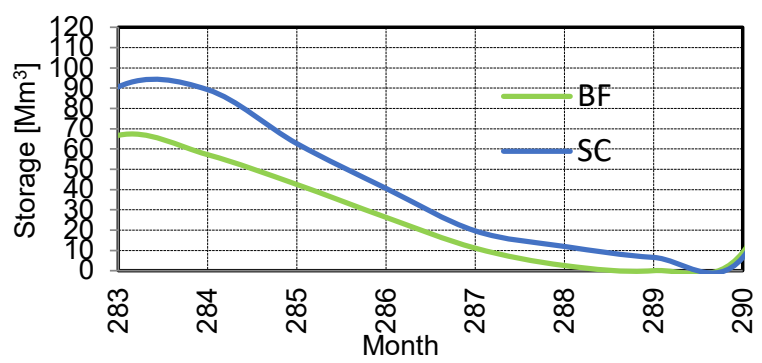

Figure 6. Simulated storage at Monte Cotugno reservoir in the months of the big failure of DSS-

$\mathrm{BF}$ for drift $=0.9$ from April (month 283) to

November (month 290) of year 24 of the simulation period

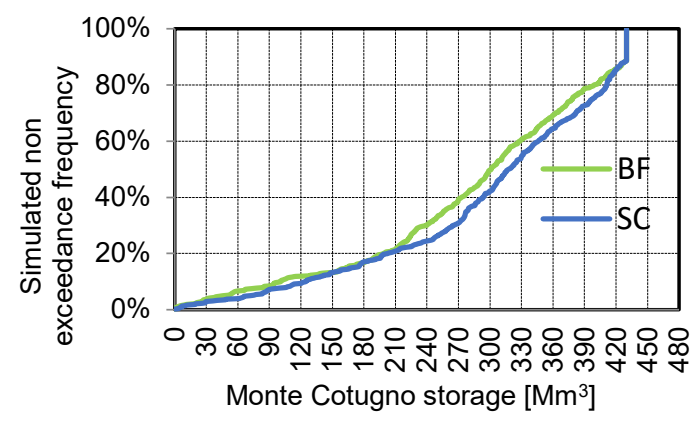

Figure 7. Simulated non-exceedance frequency of storage levels at Monte Cotugno reservoir for drift $=0.9$

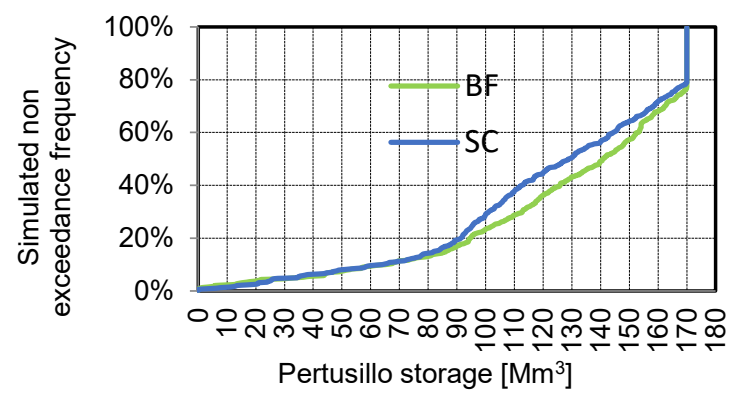

Figure 8. Simulated non-exceedance frequency of storage levels at Pertusillo reservoir for drift $=0.9$

\section{CONCLUSIONS}

The paper has presented an application of the multi-scenario DSS (DSS-SC) to a real-world situation. Results show that in terms of costs, DSS-SC compares quite favourably with the best possible option: a single scenario DSS with six-months ahead inflow forecasts coinciding with real inflows, DSS-BF. In one demand condition, described by a drift of 0.9 , DSS-SC even outperforms DSS-BF. Investigation of this behaviour led to recognize that, at least for this drift, DSS-SC would manage the system so to keep the largest reservoir of the system full enough to allow issuing less restrictive irrigation supply reductions than its DSS-BF counterpart and would therefore reduce the associated scarcity costs. This thought-provoking situation, if one the one hand confirms that in these long-memory systems "abrupt" failures can be the consequence of long-term policies and decision styles, on the other hand stimulates the reflection that DSS performances can indeed depend on a number of different factors that need to be investigated in deeper detail. From this standpoint, a single historic time series is probably not enough to explore the different possible behaviours of the DSS. For this reason, a stochastic validation of DSS-SC, by simulating its behaviour through synthetic time series, is in order and is the next research objective.

\section{REFERENCES}

Arena, C., Cannarozzo, M., Mazzola, M.R. (2017). Exploring the Potential and the Boundaries of the Rolling Horizon Technique for the Management of Reservoir Systems with over-Year Behaviour. Water Resources Management 31(3), 867-884.

Arena, C., Cannarozzo, Oliva, F., M., Mazzola, M.R. (2019). A multi-scenario Decision Support System for real-time operation of over-year multi-reservoir systems 1. Model description. Proceedings MODSIM 2019 Conference, 1-6 December 2019, Canberra, Australia.

Benaglia T., Chauveau D., Hunter D., Young D. (2009). Mixtools: An R Package for Analyzing Finite Mixture Models. Journal of Statistical Software 32 (6), 1-29.

Oliva, F. (2019). Sistema di Supporto alle Decisioni per la gestione ottimale di un sistema idrico complesso. MS Thesis, Department of Engineering, Palermo University, Italy, July 2019.

Rockafellar, R.T., Wets, R. J-B (1987). Scenario and policy aggregation in optimization under uncertainty", IIASA Working Paper WP-87-119, Laxenburg, Austria.

Vogel, R.M., Lane, M., Ravindiran, R.S., Kirshen, P. (1999). Storage reservoir behavior in the United States, Journal of Water Resources Planning and Management ASCE 125(5), 245-254. 